Troubled in the Land of Enchantment 



\section{Troubled in the Land of Enchantment}

Adolescent Experience of Psychiatric Treatment

Janis H. Jenkins and
Thomas J. Csordas

甲ㅜ 
University of California Press

Oakland, California

(C) 2020 by Thomas J. Csordas and Janis H. Jenkins

Library of Congress Cataloging-in-Publication Data

Names: Csordas, Thomas J., author. I Jenkins, Janis H., author.

Title: Troubled in the land of enchantment : adolescent experience of psychiatric treatment / Thomas J. Csordas and Janis H. Jenkins.

Description: Oakland, California : University of California Press, [2020] I Includes bibliographical references and index.

Identifiers: LCCN 202000 I 564 (print) I LCCN 202000 I 565

(ebook) I ISBN 97805203435 I I (cloth) |

ISBN 9780520343528 (paperback) I

ISBN 97805209750 I9 (epub)

Subjects: LCSH: Adolescent psychotherapy—Residential treatment-New Mexico. I Adolescent psychiatryNew Mexico. I Psychiatric hospital care-New Mexico.

Classification: LCC RJ504.5 .C78 2020 (print) I LCC RJ 504.5 (ebook) I DDC 6I6.89/I40835-dc23

LC record available at https://lccn.loc.gov/202000 I 564

LC ebook record available at https://lccn.loc .gov/202000I 565

Manufactured in the United States of America

$\begin{array}{llllllllll}29 & 28 & 27 & 26 & 25 & 24 & 23 & 22 & 21 & 20\end{array}$

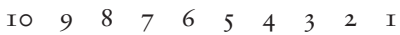

